\title{
Heterogeneous Network Access Network Selection Algorithm Based on Analytic Hierarchy Process and Improved Variable Weight
}

\author{
Cui Juanping1, Yuan Jinsha1, Chen Zhixiong1, Zhang Huale2, Han Dongsheng1 \\ 1School of Electrical and Electronic Engineering, North China Electric Power University, Baoding, China \\ 2Information \& Communication Branch, State Grid Anhui Electric Power Company, Hefei, China
}

\begin{abstract}
Aiming at the problem that the weights of constant weights cannot be changed once they are determined, this paper proposes a network access selection algorithm based on analytic hierarchy process and improved variable weight. The algorithm calculates the weights of indexes based on the analytic hierarchy process, and then introduces the improved punishment variable weight theory. According to user and traffic preferences, the indexes are prioritized. When indexes are flawed, different levels of indexes are subject to different levels of punishment. The algorithm can change the constant weight based on actual parameter values and user preferences, which makes the final decision-making result more scientific and reasonable.
\end{abstract}

\section{INTRODUCTION}

With the development of wireless communication technologies, the mobile access devices have been seen an explosive growth. The problem of network access selection has become a hot topic in the heterogeneous network (Yu, H. and Liang, G., 2017).

In the study of the existing network access selection algorithms, the analytic hierarchy process (AHP) is the most commonly used method. It can convert qualitative issues into quantitative ones, thus making the complex problems systematic and hierarchical. Liu, J. and $\mathrm{Li}, \mathrm{X}$. (2013) propose a handover decision algorithm based on AHP for WLAN/ cellular networks, which models the network handover decision problem as a fuzzy multiattribute hierarchical decision problem. Habbal, A., et al. (2017) combine the concept of context awareness with AHP to determine the index weights. This algorithm can effectively reduce the number of handover. Sui, N., et al. (2016) propose an integrated network selection scheme based on AHP and evolutionary game theory, which can effectively achieve load balancing.

However, the commonly used weight calculation methods only consider the relative importance of the indexes, and do not consider the actual measurement values of the indexes. The weight cannot be changed once it is determined which is unreasonable. Therefore, the punishment variable weight theory (Cai, W., et al., 2017) is introduced in this paper, and we have made some improvements to it.
This paper proposes a network access selection algorithm based on AHP and improved variable weight. Calculate the weight of indexes by AHP, and prioritize indexes based on user preferences and business requirements. Then calculate the variable weights according to the actual parameters and the priority of the indexes. Finally, calculate the network comprehensive score and obtain the best target network. The algorithm not only changes the weight of the index with the change of parameters, but also takes into account user preferences and traffic requirements when changing weights, which makes the decision more scientific and reasonable.

\section{ACCESS SELECTION ALGORITHM BASED ON AHP AND IMPROVED VARIABLE WEIGHT}

\subsection{HETEROGENEOUS NETWORK SYSTEM MODEL}

The heterogeneous network system model studied in this paper consists of three wireless networks: LTE, WiMAX, and WiFi, as shown in Figure 1. It is known that the physical layers of these three types of wireless networks employ OFDM modulation technology, and the mobile terminal has multiple interfaces, which can access any one of the networks and provide services for users anytime and anywhere.

\footnotetext{
*Corresponding author:

CuiJP0708@163.com, yuanjinsa@1260com, chenzx1983@sohu.com, 


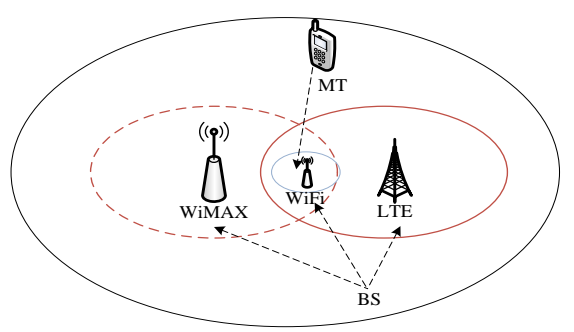

Figure 1. Heterogeneous network system model.

This paper selects six (ie, $m=6$ ) relatively typical decision indexes, namely: received signal strength, cost, delay, network load, packet loss rate and data rate.

\subsection{DETERMINE THE CONSTANT WEIGHT OF THE INDEX BASED ON AHP}

AHP is suitable for the complex systems with multiple levels and multiple objectives. The specific steps are as follows (Liu, J. and Li, X., 2013):

Step 1 Establish a pairwise matrix.

According to the Saatys scale of importance, the pairwise matrix $D$ can be obtained, as shown in equation (1):

$$
D=\left[\begin{array}{ccc}
d_{11} & \cdots & d_{1 m} \\
\cdots & \cdots & \cdots \\
d_{m 1} & \cdots & d_{m m}
\end{array}\right]
$$

where $d_{i j}(i, j=1,2, \ldots, m)$ represents the importance of the $i$ th index compared to the $j$-th index and $d_{i i}=1$, $d_{j i}=1 / d_{i j}$.

Step 2 Calculate the weight of the index and check the consistency of the pairwise matrix.

The index weight $W$ can be expressed by (2)

$$
W=\left[\begin{array}{c}
w_{1} \\
\vdots \\
w_{m}
\end{array}\right], w_{j}=\frac{\sum_{j=1}^{m}\left(d_{i j} / \sum_{i=1}^{m} d_{i j}\right)}{m}
$$

The experts' understanding is uncertain, so we need to check the consistency of the pairwise matrix. The consistency check index $C I$ is shown in (3):

$$
C I=\frac{\lambda_{\max }-m}{m-1}, \lambda_{\max }=\frac{1}{m} \sum_{j=1}^{m} \frac{(D w)_{j}}{w_{j}}
$$

where the closer the $C I$ is to 0 , the better the consistency of the pairwise matrix is. In order to appropriately reduce the consistency requirement, the average random consistency index $R I$ is introduced to modify the $C I$. Table 1 shows the values of the $R I$.

The revised consistency indicator is $C R=C I / R I$. And under normal circumstances, it is assumed that the consistency indicator should satisfy $\mathrm{CR}<0.05$; otherwise, it returns to Step 1.
Table 1. Values of RI corresponding to different dimensions of the matrix.

\begin{tabular}{|c|c|c|c|c|c|c|c|}
\hline dimension & 1 & 2 & 3 & 4 & 5 & 6 & 7 \\
\hline RI & 0 & 0 & 0.58 & 0.96 & 1.12 & 1.24 & 1.32 \\
\hline
\end{tabular}

\subsection{ACCESS SELECTION ALGORITHM BASED ON IMPROVED VARIABLE WEIGHT}

For the weights of the indexes calculated by AHP, once they are determined, it cannot be changed. When a certain index has a defect, it will result in a decrease in network performance. So this paper introduces the punishment variable weight theory. Specific steps are as follows:

Step 1 Normalize the network parameters.

For profitable indexes

$$
x_{i j}=\frac{x_{i j}^{0}-\min _{j}\left(x_{i j}^{0}\right)}{\max _{j}\left(x_{i j}^{0}\right)-\min _{j}\left(x_{i j}^{0}\right)}
$$

For cost indexes

$$
x_{i j}=\frac{\max _{j}\left(x_{i j}^{0}\right)-x_{i j}^{0}}{\max _{j}\left(x_{i j}^{0}\right)-\min _{j}\left(x_{i j}^{0}\right)}
$$

where $i=1,2, \ldots, n ; j=1,2, \ldots, m ; \quad x_{i j}$ is the value of the normalized index; $x_{i j}^{0}$ denotes the initial value of the $j$-th decision index in the $i$-th candidate network; $\max _{j}\left(x_{i j}^{0}\right)$ and $\min _{j}\left(x_{i j}^{0}\right)$ denote the maximum and minimum values of the $j$-th index of the candidate network, respectively.

Step 2 Calculate variable weight of index.

The variable weight can be obtained by (6)

$$
w_{i j}^{V}=\frac{w_{j}\left(x_{i j}\right)^{\alpha-1}}{\sum_{j=1}^{6} w_{j}\left(x_{i j}\right)^{\alpha-1}}
$$

where $w_{i j}^{V}$ denotes the variable weight calculated by variable weight function; when $\alpha \in[0,1]$, the index weight decreases monotonically with $x_{i j}$

When $\alpha$ takes different values, the punishment for the index is also different. We assume that the load of TLE network is very high, while little change in other index values. The constant weights obtained by AHP are $w_{R S S}=0.0932, w_{\text {Cost }}=0.1947, w_{\text {Rate }}=0.1045, w_{\text {Packet }}=0.2045$, $w_{\text {Delay }}=0.2069$ and $w_{\text {Load }}=0.1962$. The changes in the weight of each index are shown in Figure 2. 


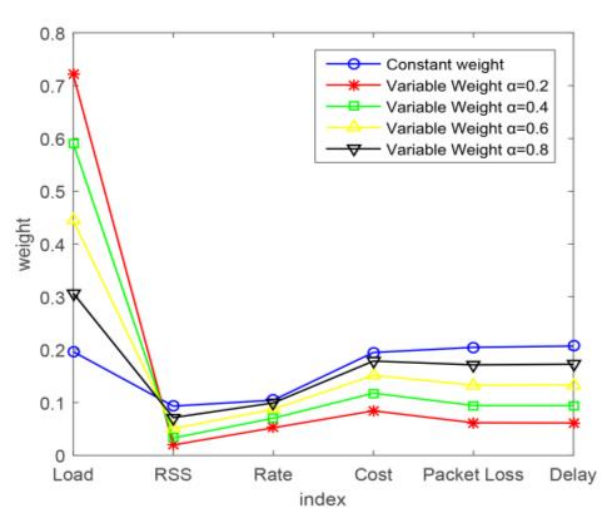

Figure 2. The changes of index weights.

It can be seen that the larger the value of $\alpha$, the smaller the adjustment of the index weight by variable weight function. When $\alpha=1$, the index weight is equal to the constant weight.

Due to differences in user's preferences and traffic requirements, we have made improvements to the punishment variable weight. First, the indexes can be prioritized based on user preferences and traffic requirements. Then according to the priority, a value of $\alpha$ is set for each index. The higher the priority is, the smaller the value of $\alpha$ is.

Thus, formula (6) is corrected, and the variable weight of the index is obtained by formula (7),

$$
w_{i j}^{I V}=\frac{w_{j}\left(x_{i j}\right)^{\alpha_{j}-1}}{\sum_{j=1}^{6} w_{j}\left(x_{i j}\right)^{\alpha_{j}-1}}
$$

where $w_{i j}^{I V}$ denotes the variable weight calculated by improved punishment variable weight function; $\alpha_{j}$ represents the value of $\alpha$ for the $j$-th index and $\alpha_{j} \in[0,1]$.

Step 3 Get the conclusion of comprehensive evaluation.

The network comprehensive score is calculated by the following formula,

$$
p_{i}=\sum_{j=1}^{m} w_{i j}^{I V} x_{i j}
$$

where $p_{i}(i=1,2, . ., n)$ represents the composite score of the $i$-th candidate network. So the candidate networks are sorted in descending order, and the top ranked network is selected for access.

\section{SIMULATION RESULTS AND ANALYSIS}

A simulation analysis of the heterogeneous network system model constructed in Figure 1 is performed. Assume that in the overlapping coverage areas of these three networks, the new service is reached by Poisson distribution. The simulation parameters of each network are shown in Table 2 (Habbal, A., et al., 2017; NguyenVuong, Q. T., et al., 2013).

Table 2. Network simulation parameters.

\begin{tabular}{|c|c|c|c|}
\hline $\begin{array}{c}\text { Network } \\
\text { type }\end{array}$ & LTE & WiMAX & WiFi \\
\hline Fc(Ghz) & 2.0 & 2.3 & 2.4 \\
\hline $\begin{array}{c}\text { Coverage } \\
\text { zone }(\mathrm{m})\end{array}$ & 1000 & 1000 & 100 \\
\hline $\begin{array}{c}\text { Cost } \\
\text { (Cent/Mbps) }\end{array}$ & {$[4,8]$} & {$[3,7]$} & {$[1,3]$} \\
\hline $\begin{array}{c}\text { Data rate } \\
\text { (Mbps) }\end{array}$ & {$[100,300]$} & {$[50,250]$} & {$[50,200]$} \\
\hline Delay(ms) & {$[80,100]$} & {$[80,150]$} & {$[100,150]$} \\
\hline $\begin{array}{c}\text { Packet loss } \\
\left.\text { (per } 10^{6}\right)\end{array}$ & {$[10,15]$} & {$[10,17]$} & {$[10,20]$} \\
\hline
\end{tabular}

In order to verify the performance of the network selection algorithm proposed in this paper, we compare it with the AHP algorithm using the MATLAB simulation software.

Assume that a user is very valuing the price of service and there are no special requirements for other indexes. When both the data rate and cost of the LTE network have defects, Figure 3 shows the change of the weight of the indexes, where $\alpha=0.2$ is set in the punishment variable weight algorithm and $\alpha=[0.4,0.4,0.4,0.2,0.4,0.4]$ in the improved variable weight algorithm.

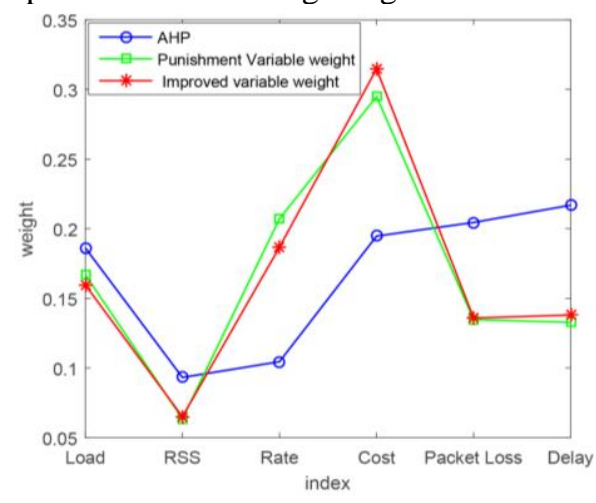

Figure 3. The changes of index weights.

It can be seen that the algorithm based on improved variable weight can make the weight of index change with the changes of the actual index parameters. When the index is abnormal, the weight increases. And compared with the algorithm based on punishment variable weight, the algorithm is able to make different penalties for indexes, according to the different user preferences. 


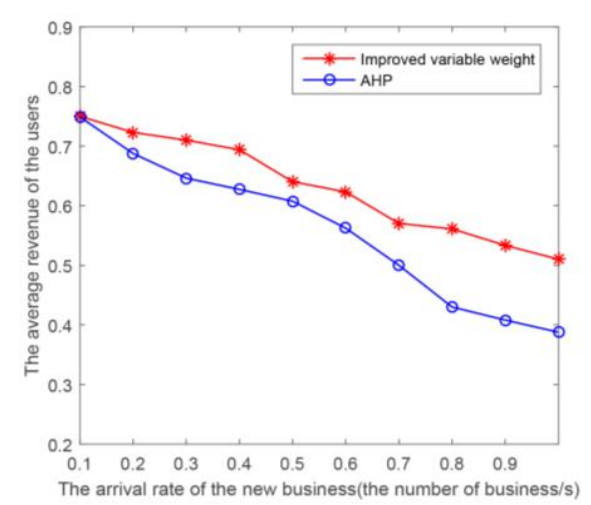

Figure 4. The average revenues of the users.

Figure 4 shows the relationship between the average revenue of the users and the arrival rate of the new business. With the increase of the arrival rate of new business, the average revenues of the users show a downward trend, but the average revenue of the users calculated by the algorithm based on improved variable weight is obviously higher than the other. This is because with the continuous access of new services, the network resources are gradually decreasing. At the same time, the algorithm based on improved variable weight takes full consideration of the actual measurement value and user's preferences. So it can guarantee the revenue of the users.

\section{CONCLUSIONS}

The paper proposes a network access selection algorithm based on analytic hierarchy process and improved variable weight. This algorithm uses AHP method to calculate the constant weight, and introduces the improved punishment variable weight theory. According to the user's preferences, the indexes are prioritized. Different levels of indexes have different punishment when the indexes are flawed. So the algorithm can make the weights change. Therefore, the final judgment result is more scientific and reasonable.

\section{ACKNOWLEDGMENTS}

This work was supported by the National Natural Science Foundation of China (No.61601182, No.61771195), Natural Science Foundation of Hebei Province (No.F2017502059, No.F2018502047), and the Fundamental Research Funds for the Central Universities( No.2017MS109).

\section{REFERENCES}

1. Cai, W., Liu, W., et al., 2017. Analysis of Electromagnetic Loop Network on Loop Closing or Opening Based on Model of Optimal Comprehensive Evaluation With Punitive Variable Weight. Power System Technology, 41(7), 23162323.

2. Habbal, A., Goudar, S. L., et al., 2017. ContextAware Radio Access Technology Selection in 5G
Ultra Dense Networks. IEEE Access, 5(99), 66366648.

3. Liu, J., Li, X., 2013. Handover algorithm for WLAN/cellular networks with analytic hierarchy process. Journal on Communications, 34(2), 6572.

4. Nguyen-Vuong, Q. T., Agoulmine, N., et al., 2013. Multicriteria optimization of access selection to improve the quality of experience in heterogeneous wireless access networks. IEEE Transactions on Vehicular Technology, 62(4), 1785-1800.

5. Sui, N., Zhang, D., et al., 2016. Network selection for heterogeneous wireless networks based on multiple attribute decision making and Evolutionary Game Theory. 25th Wireless and Optical Communication Conference. IEEE, 1-5.

6. Yu, H., Liang, G., 2017. A survey of access selection algorithms in heterogeneous wireless networks. Journal of Harbin Institute of Technology, 49(11), 178-188. 